\title{
BMJ Open Rationale and design of the IRON-AF study: a double-blind, randomised, placebo-controlled study to assess the effect of intravenous ferric carboxymaltose in patients with atrial fibrillation and iron deficiency
}

Samuel J Tu (D) , Adrian D Elliott, Nicole Hanna-Rivero, Celine Gallagher (1) , Ricardo S Mishima, Ellen Lyrtzis, Danielle Wlochowicz, Nicholas AR Clarke, Kurt C Roberts-Thomson, Michael B Stokes, Mehrdad Emami, Dennis H Lau, Prashanthan Sanders (D), Christopher X Wong

To cite: Tu SJ, Elliott AD, HannaRivero N, et al. Rationale and design of the IRON-AF study: a double-blind, randomised, placebo-controlled study to assess the effect of intravenous ferric carboxymaltose in patients with atrial fibrillation and iron deficiency. BMJ Open 2021;11:e047642. doi:10.1136/ bmjopen-2020-047642

- Prepublication history for this paper is available online. To view these files, please visit the journal online (http://dx.doi. org/10.1136/bmjopen-2020047642).

Received 07 December 2020 Accepted 05 July 2021

Check for updates

(c) Author(s) (or their employer(s)) 2021. Re-use permitted under CC BY-NC. No commercial re-use. See rights and permissions. Published by BMJ.

Centre for Heart Rhythm Disorders, The University of Adelaide and Royal Adelaide Hospital, Adelaide, South Australia, Australia

Correspondence to A/Prof Christopher X Wong; c.wong@adelaide.edu.au

\section{ABSTRACT}

Introduction Atrial fibrillation (AF) is associated with significantly impaired quality-of-life. Iron deficiency (ID) is prevalent in patients with AF. Correction of ID in other patient populations with intravenous iron supplementation has been shown to be a safe, convenient and effective way of improving exercise tolerance, fatigue and quality-of-life. The IRON-AF (Effect of Iron Repletion in Atrial Fibrillation) study is designed to assess the effect of iron repletion with intravenous ferric carboxymaltose in patients with $\mathrm{AF}$ and ID.

Methods and analysis The IRON-AF study is a doubleblind, randomised controlled trial that will recruit at least 84 patients with AF and ID. Patients will be randomised to receive infusions of either ferric carboxymaltose or placebo, given in repletion and then maintenance doses. The study will have follow-up visits at weeks 4,8 and 12. The primary endpoint is change in peak oxygen uptake from baseline to week 12, as measured by cardiopulmonary exercise testing (CPET) on a cycle ergometer. Secondary endpoints include changes in quality-of-life and AF disease burden scores, blood parameters, other CPET parameters, transthoracic echocardiogram parameters, 6-minute walk test distance, 7-day Holter/Event monitor burden of AF, health resource utilisation and mortality.

Ethics and dissemination The study protocol has been approved by the Central Adelaide Local Health Network Human Research Ethics Committee, Australia. The results of this study will be disseminated through publications in peer-reviewed journals and conference presentations. Trial registration number Australian New Zealand Clinical Trials Registry (ACTRN12620000285954).

\section{INTRODUCTION}

Atrial fibrillation (AF) is the most common sustained arrhythmia faced in clinical practice, and is independently associated with
Strengths and limitations of this study

- This double-blind, randomised, placebo-controlled trial will provide clinically relevant evidence on the effect of iron repletion with intravenous ferric carboxymaltose on exercise tolerance in patients with atrial fibrillation (AF) and iron deficiency, as measured by cardiopulmonary exercise testing.

- Other clinically important endpoints will be assessed, including quality-of-life and disease burden assessed by questionnaires, changes to blood and echocardiogram parameters, 6-minute walk test distance and AF burden on 7-day Holter/Event monitoring.

- Iron deficiency will be defined according to the definition used in the clinical trials of iron repletion in heart failure patients: serum ferritin $<100 \mu \mathrm{L}$ or serum ferritin $<300 \mu \mathrm{L}$ with transferrin saturation $<20 \%$.

- The IRON-AF trial will provide the first assessment on the potential efficacy of iron repletion in patients with AF. Though objective exercise testing will be performed, study findings pertaining to improvements in symptoms will be limited by self-reported questionnaires; demonstration of a benefit in terms of hospitalisations would require a larger sample size and longer duration of follow-up.

an increased risk of stroke, heart failure and death. ${ }^{1}$ Hospitalisations for AF have been exponentially increasing, and the disease represents a growing burden on the healthcare systems worldwide. ${ }^{2-4}$ This increasing trend is likely multifactorial, fuelled by the growing burden of cardiometabolic risk factors such as obesity, an ageing population, 
as well as recurrent admissions due to medical complications and recurring symptoms. ${ }^{5}$

Individuals with AF have an impaired functional status and quality-of-life (QoL) that is widely affected across domains of physical and social functioning, mental and general health. ${ }^{67}$ One of the cardinal symptoms of AF is reduced exercise capacity, and this occurs regardless of the presence of heart failure. ${ }^{8-12}$ Other prominent symptoms such as dyspnoea and fatigue are also associated with a reduction in functional status, and a greater burden of AF symptoms is negatively correlated with QoL. ${ }^{13}$ Despite the symptomatic benefits that cardioversion and catheter ablation may provide, ${ }^{1415}$ recurrences are common. There is thus a need for novel treatments to improve the symptomatology of patients with $\mathrm{AF}$, beyond the pillars of rate control, rhythm control and risk factor management. ${ }^{16}$ Treatments targeting exercise intolerance in particular as a therapeutic goal may bring improvements to symptoms and QoL, ${ }^{17}$ and may also encourage greater engagement with risk factor management. Epidemiological studies also have shown poor cardiorespiratory fitness in patients with $\mathrm{AF}$ to be a predictor of arrhythmia recurrence, ${ }^{18} 19$ as well as all-cause and cardiovascular mortality. ${ }^{20}$

Iron plays an important role in cardiorespiratory fitness and is essential in the uptake, transport, storage and utilisation of oxygen through haem, and in various iron-dependent oxidative enzymes and processes such as neurotransmitter synthesis, protein synthesis and organogenesis. ${ }^{21}$ Iron deficiency (ID) that leads to anaemia impairs physical work performance most evidently through a reduction in oxygen transport, the most important determinant of maximal oxygen consumption $\left(\mathrm{VO}_{2 \max }\right)$. However, ID even in the absence of anaemia may attenuate exercise performance, owing to the reduced activity of iron-dependent oxidative enzymes that are particularly important in the ability to maintain prolonged submaximal exercise. ${ }^{22}{ }^{23}$ In observational studies of heart failure patients, ID independent of the presence of anaemia is associated with reduced $\mathrm{VO}_{2 \text { peak }}$ and high minute ventilation/carbon dioxide production $\left(\mathrm{V}_{\mathrm{E}} / \mathrm{VCO}_{2}\right)$ slope. ${ }^{2425} \mathrm{ID}$ has also been shown to be a stronger predictor of poorer QoL in these patients than the presence of anaemia. ${ }^{26} 27$

ID that leads to anaemia warrants treatment with iron supplementation, but the role of iron supplementation in non-anaemic ID is less established. In the younger and healthy population, improvements in fatigue but not exercise tolerance may be seen. ${ }^{28}$ In patients with heart failure with reduced ejection fraction (HFrEF) and concurrent ID, randomised controlled trials of intravenous iron repletion have shown significant improvements in symptoms, exercise tolerance, QoL and heart failure hospitalisations, regardless of the presence of anaemia. ${ }^{29-31}$ Importantly, these trials recruited participants using a definition of functional ID, reflecting a state of maldistribution of iron in the body seen in chronic inflammatory states. Ferritin is non-specifically elevated as an acute-phase reactant with inflammation due to increased hepcidin production and subsequent inhibition of ferroportin, leading to impaired absorption and utilisation of iron. To account for this, functional ID has previously been defined as a serum ferritin $<100 \mu \mathrm{L}$, or serum ferritin $<300 \mu \mathrm{L}$ with transferrin saturation (Tsat) $<20 \%$. Using this definition, a recent retrospective study examining patients with nonvalvular $\mathrm{AF}$ without heart failure demonstrated that ID was present in $48 \%$ of patients. ${ }^{32}$

Further investigation into haematinic deficiencies in AF is likely worthwhile for two reasons: first, the pathophysiology of $\mathrm{AF}$ and $\mathrm{HF}$ are closely interlinked, and inflammation has been implicated in both pathological processes. ${ }^{33}$ Second, anaemia is a common comorbidity in individuals with AF, present in approximately $16 \%$ of patients. ${ }^{34}$ Potential explanations for both anaemia and ID in these patients include chronic inflammation and an increased risk of clinically evident or occult bleeding with the use of oral anticoagulants. Of note, observational data demonstrate that anaemia is independently associated with increased mortality, stroke/systemic thromboembolism and bleeding in patients with $\mathrm{AF}^{34}$

Iron supplementation in patients with $\mathrm{AF}$ and comorbid ID has not been studied previously but may be a simple and convenient way to potentially provide symptomatic relief from fatigue and exercise intolerance. Thus, the IRON-AF (Effect of Iron Repletion in Atrial Fibrillation) trial was designed to assess the effect of the supplementation of iron with intravenous ferric carboxymaltose (FCM) on exercise tolerance, fatigue, QoL and other parameters in patients with $\mathrm{AF}$ and ID.

\section{METHODS AND ANALYSIS}

The IRON-AF study is an investigator-initiated, doubleblind, randomised, placebo-controlled trial. The overall study design is outlined in figure 1 , and the study inclusion and exclusion criteria are listed in box 1 . The key inclusion criteria are (1) paroxysmal or persistent AF; (2) serum ferritin $<100 \mu \mathrm{L}$, or serum ferritin $<300 \mu \mathrm{L}$ with Tsat $<20 \%$; and (3) haemoglobin (Hb) $<150 \mathrm{~g} / \mathrm{L}$.

\section{Baseline testing}

After obtaining written informed consent and confirming eligibility, patients will have baseline assessments performed, including medical history (including comorbidities and medications) and anthropomorphic measures, health questionnaires (including a fatigue score, the Atrial Fibrillation Severity Scale [AFSS], the 36-Item Short Form Survey [SF-36] and the Atrial Fibrillation Effect on Quality-of-Life Questionnaire [AFEQT]), resting transthoracic echocardiogram (TTE), 6-minute walk test (6MWT), cardiopulmonary exercise testing (CPET) and a 7 day Holter/Event monitor. Participants will undergo randomisation following completion of all baseline tests.

\section{Study randomisation}

Participants will be randomised using a computergenerated randomisation schedule in a 1:1 ratio to either 


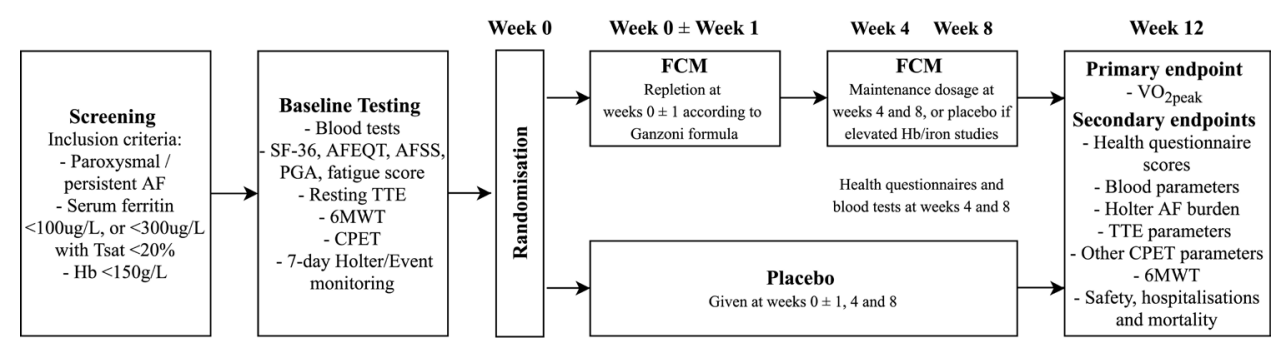

Figure 1 Schema of the IRON-AF trial. 6MWT, 6-minute walk test; AF, atrial fibrillation; AFEQT, Atrial Fibrillation Effect on Quality-of-Life Questionnaire; AFSS, Atrial Fibrillation Severity Scale; CPET, cardiopulmonary exercise testing; FCM, ferric carboxymaltose; Hb, haemoglobin; PGA, patient global assessment; QoL, quality-of-life; SF-36, 36-Item Short Form Survey; Tsat, transferrin saturation; TTE, transthoracic echocardiogram; $\mathrm{VO}_{2 \text { peak }}$, peak oxygen uptake.

active treatment or placebo using permutated blocks of random sizes. We anticipate that a number of recruited participants will be concurrently involved in our AF risk factor management clinics involving weight and physical activity counselling, ${ }^{35}$ and so randomisation will be stratified according to baseline participation in these clinics.

\section{Study drug and blinding}

The active treatment is FCM (Ferinject, Vifor Pharma, Australia), to be delivered intravenously as an undiluted bolus dose, over $15 \mathrm{~min}$ for doses $\geq 500 \mathrm{mg}$ or at a rate of $100 \mathrm{mg} / \mathrm{min}$ for doses $<500 \mathrm{mg}$. The placebo is $0.9 \%$ sodium chloride, to be delivered intravenously at the equivalent rate. FCM is a dark brown solution and therefore easily distinguishable from placebo. Hence, to maintain subject blinding, both the study drug and placebo will be delivered with the participant's arm behind an opaque curtain.

For subjects in the active treatment group, the dose of FCM for repletion will be calculated using the Ganzoni formula shown in figure 2. During week 0 , subjects in the active treatment group will receive FCM, up to a maximum dose of $1000 \mathrm{mg}$. Should the dose for repletion not be completely delivered then, participants will return during week 1 to receive the remainder of the dose.

At the start of weeks 4 and 8 , subjects will have iron studies performed. The results of these studies will be reviewed by an unblinded study coordinator not involved in assessments of efficacy or safety. Subjects will then return to the study centre at the end of weeks 4 and 8 and receive either a maintenance dose of $200 \mathrm{mg}$ FCM or placebo, according to treatment group. In the active treatment group, in cases of elevated levels of $\mathrm{Hb}>160$ $\mathrm{g} / \mathrm{L}$, ferritin $>800 \mu \mathrm{g} / \mathrm{L}$ or ferritin $>500 \mu \mathrm{g} / \mathrm{L}$ when Tsat $>50 \%$, placebo will be given instead of FCM.

\section{Study end point and assessments}

The primary, secondary and safety endpoints are listed in box 2. Clinical assessments for efficacy and safety will be completed at follow-up visits in week 4 , week 8 , and the final visit in week 12. All study coordinators involved in performing clinical assessments will be blinded to participant's group allocation. The primary efficacy assessment for $\mathrm{VO}_{\text {2peak }}$ will occur in week 12 derived by CPET.
CPET will be conducted using an electromagnetically controlled cycle ergometer (Corvial, Lode, The Netherlands) with a 1 min ramp protocol to volitional exhaustion. Twelve-lead ECG (Vyntus ECG, Vyaire, USA) will be monitored to record heart rate and rhythm throughout rest, exercise and recovery. Pulmonary gas exchange will be recorded continuously through a metabolic cart (Vyntus CPX, Vyaire, USA). Resting measures will be obtained during a minimum of 2 min seated rest. A minimum of 2 min recovery will be commenced after participants attained peak exercise. The anaerobic threshold will be determined using the V-slope method. $\mathrm{VO}_{2 \text { peak }}$ will be taken as the highest 20 second averaged sample of $\mathrm{VO}_{2}$ during peak exercise, and predicted values of $\mathrm{VO}_{2 \text { peak }}$ will be obtained using the Wasserman and Hansen equations. ${ }^{36} \mathrm{~V}_{\mathrm{E}} / \mathrm{VCO}_{2}$ will be calculated by using a linear regression slope of minute ventilation and $\mathrm{CO}_{2}$ output between rest and peak exercise.

Resting transthoracic echocardiography (Vivid 7, GE Healthcare, Norway) will be performed at baseline and week 12. Transthoracic images will be obtained in the apical four-chamber, three-chamber and two-chamber views, and parasternal long and short axis views. Echocardiographic variables, including systolic and diastolic function, atrial volumes and global longitudinal strain, will be collected and analysed by a sonographer, and subsequently analysed by a cardiologist blinded to the randomisation.

Blood parameters collected will include a complete blood count, iron studies (including serum iron, ferritin, transferrin and transferrin saturation), electrolytes, urea, creatinine, liver function tests, C-reactive protein, erythrocyte sedimentation rate and NT-proB-type natriuretic peptide. Values will be obtained at baseline, weeks 4, 8 and 12 , with venesection performed prior to infusions and/or assessments. If a significant reduction in haemoglobin is detected during the course of monitoring, the participant's treating physicians will be informed and a decision to withdraw from the study will be made in conjunction with the participant's wishes.

Validated generic and disease-specific QoL questionnaires, including the SF-36 and AFEQT, as well as a validated disease-specific symptom questionnaire, the AFSS, will be used to characterise changes to patient well-being 
Box 1 Inclusion and exclusion criteria for the IRON-AF (Effect of Iron Repletion in Atrial Fibrillation) trial

\section{Inclusion criteria}

- At least 18 years of age

- Paroxysmal or persistent AF

- Stable medical therapy in the 4 weeks prior to enrolment with no dose changes of AF drugs in the last 2 weeks

- Haemoglobin $<150 \mathrm{~g} / \mathrm{L}$

- Serum ferritin $<100 \mu \mathrm{g} / \mathrm{L}$ or serum ferritin $<300 \mu \mathrm{g} / \mathrm{L}$ if Tsat $<20 \%$

\section{Exclusion criteria}

- Known, or detected on screening, deficiency of vitamin B12 or serum folate

- Known hypersensitivity to parenteral iron preparations

- History of primary or secondary haemochromatosis

- Known haemoglobinopathy or haemolytic anaemia

- History of erythropoietin-stimulating agent, intravenous iron therapy and/or blood transfusion in the 6 weeks prior to randomisation

- Requiring treatment with blood transfusion in the next 3 months

- Requiring surgery with anticipated moderate or severe blood loss in the next 3 months

$>$ Ongoing blood loss

- Oral iron therapy at doses $>80 \mathrm{mg} /$ day (of elemental iron) in the 1 week prior to randomisation (ongoing use of multivitamins containing $\leq 80 \mathrm{mg} /$ day is permitted)

- Cardioversion, catheter ablation, AV nodal ablation with pacemaker insertion or surgical ablation within the past 3 months, or planned within the next 3 months

- Body weight $\leq 35 \mathrm{~kg}$

- Exercise training programme(s) in the 3 months prior to screening, or planned in the next 3 months

- Current active autoimmune or systemic inflammatory diseases, or systemic infection or bacteraemia

- Screening AST/ALT above three times the upper limit of normal range

- Known chronic liver disease, hepatitis B or hepatitis C infection

- Known active malignancy, with the exception of squamous cell and basal cell carcinoma of the skin, and cervical intraepithelial neoplasia

- Currently receiving systemic chemotherapy and/or radiotherapy

- Known chronic kidney disease or history of dialysis, current dialysis or dialysis planned within the next 3 months

- Currently pregnant, breast feeding or trying to conceive within the next 3 months

- Acute myocardial infarction, transient ischaemic attack, or stroke within the last 3 months prior to randomisation

- Coronary artery bypass graft, percutaneous intervention or major surgery (including thoracic and cardiac surgery) within the last 3 months (diagnostic catheterisations are allowed)

- Subject is unable to perform exercise testing, either because of investigator judgement or due to comorbidities severely impacting exercise capacity (including, but not limited to: severe valvular disease, severe obstructive lung disease, unstable angina)

- Subject is currently enrolled in, or is within 30 days of completion of, another interventional study

- Subject has previously been randomised to this study (subjects may be rescreened if they previously did not meet the eligibility criteria)

- Subject will not be available for completion of all assessments as per the study protocol

AF, atrial fibrillation; ALT, alanine aminotransferase; AST, aspartate aminotransferase; AV, atrioventricular; Tsat, transferrin saturation.
Iron deficit $(\mathrm{mg})=$ body weight $(\mathrm{kg})^{*} \times\left[150^{\dagger}-\operatorname{actual} \mathrm{Hb}(\mathrm{g} / \mathrm{L})\right] \times 0.24^{\ddagger}+500^{\S}$

" IBW is to be used in patients with a BMI $>25$. For females, IBW $=45.5 \mathrm{~kg}+0.9 \mathrm{~kg} / \mathrm{cm}$ for each $\mathrm{cm}$ over $152 \mathrm{~cm}$. For males, $\mathrm{IBW}=50 \mathrm{~kg}+0.9 \mathrm{~kg} / \mathrm{cm}$ for each $\mathrm{cm}$ over $152 \mathrm{~cm}$

Target $\mathrm{Hb}$ of $150 \mathrm{~g} / \mathrm{L}$

Factor $0.24=0.0034$ (iron content of $\mathrm{Hb}$ ) $\times 0.07$ (blood volume of body weight) $\times 1000$ (conversion from $\mathrm{g} / \mathrm{L}$ to $\mathrm{mg} / \mathrm{L}$ )

Iron depot of $500 \mathrm{mg}$

Figure 2 Ganzoni formula, used to calculate the repletion dosage of ferric carboxymaltose in the IRON-AF (Effect of Iron Repletion in Atrial Fibrillation) trial. BMI, body mass index; Hb, haemoglobin; IBW, ideal body weight.

and functional status. A patient global assessment score will be obtained from responses to the question 'How do you feel about your life at the present time?', given as a

Box 2 Primary, secondary and safety endpoints of the IRON-AF (Effect of Iron Repletion in Atrial Fibrillation) trial

\section{Primary endpoints}

- Change in $\mathrm{VO}_{2 \text { peak }}(\mathrm{mL} / \mathrm{min} / \mathrm{kg})$ from baseline to week 12

\section{Secondary endpoints}

- Change in SF-36 subscale scores from baseline to weeks 4, 8 and 12

- Change in AFEQT overall and subset scores from baseline to weeks 4,8 and 12

- Change in AFSS severity and burden scores from baseline to weeks 4,8 and 12

- Change in PGA score from baseline to weeks 4, 8 and 12

- Change in fatigue score from baseline to weeks 4,8 and 12

- Change in NYHA classification from baseline to weeks 4, 8 and 12

- Changes in laboratory parameters (including $\mathrm{Hb}$, serum ferritin, iron, Tsat, NT-proBNP, CRP and ESR) from baseline to weeks 4, 8 and 12

- Changes in other CPET parameters (including ventilatory efficiency, $\mathrm{VO}_{2}$ at $\mathrm{AT}, \mathrm{HR}$ at peak and $\mathrm{AT}$, and peak oxygen pulse) from baseline to week 12

- Changes in TTE parameters (including left atrial and ventricular dimensions and volumes, diastolic function and ejection fractions) from baseline to week 12

- Change in 6MWT distance from baseline to week 12

- Change in 7-day Holter monitor-measured AF burden from baseline to week 12

- Hospitalisations (total, cardiovascular, heart failure, AF)

- Mortality (total, cardiovascular, AF)

\section{Safety endpoints}

- Hospitalisations and deaths

- Episodes of chest pain or hypotension during CPET

- Anaphylaxis or episodes of hypotension during or at completion of treatment infusion

- Episodes of serum ferritin $>1000 \mu \mathrm{g} / \mathrm{L}$

- Episodes of haemoglobin $<70 \mathrm{~g} / \mathrm{L}$

- Episodes of elevated ALT, AST, urea and creatinine

6MWT, 6-minute walk test; AF, atrial fibrillation; AFEQT, Atrial Fibrillation Effect on Quality-of-Life Questionnaire; AFSS, Atrial Fibrillation Severity Scale; ALT, alanine aminotransferase; AST, aspartate aminotransferase; AT, anaerobic threshold; CPET, cardiopulmonary exercise testing; CRP, C-reactive protein; ESR, erythrocyte sedimentation rate; $\mathrm{Hb}$, haemoglobin; HR, heart rate; NTproBNP, NT-proB-type natriuretic peptide; NYHA, New York Heart Association; PGA, patient global assessment; SF-36, 36-Item Short Form Survey; Tsat, transferrin saturation; TTE, transthoracic echocardiogram; $\mathrm{VO}_{2}$, oxygen consumption; $\mathrm{VO}_{2 \text { peak }}$, peak oxygen uptake. 
scale of 1 ('worst possible life') to 10 ('best possible life'). This question is present on the AFSS but is not included in the calculation of the AFSS severity and burden scores. A fatigue score will also be included, given as a 10-point numeric rating scale ranging from 1 ('no fatigue at all') to 10 ('very severe fatigue'). These questionnaires will be given to patients at baseline, weeks 4,8 and 12, and summary scores will be calculated in accordance with established scoring criteria.

The 6MWT will be performed at baseline and week 12 in accordance with a standardised protocol, in an area equipped with cardiopulmonary resuscitation facilities. Tests will be performed along a flat, straight route with a hard surface $32 \mathrm{~m}$ in length. Subjects will be instructed to walk the length of the course at their own pace and informed that the test will conclude after $6 \mathrm{~min}$ or if participants stopped, whichever occurs first. Coordinators will give verbal encouragements and call out the time every minute. The total distance achieved during the test, to the nearest metre, will be recorded.

Seven-day Holter/Event monitors (Spiderflash, Microport CRM, France) will be fitted onto participants at baseline and week 12, and participants will be instructed on their use. In accordance with device capabilities, a continuous Holter ECG will be recorded during the first 25 hours, before the monitor switches to its event monitor function, where only periods of R-R instability $\geq 30 \mathrm{~s}$ will be recorded. AF burden will be defined as the percentage of time spent in AF during the 7-day monitoring period.

Hospitalisation and mortality information will be obtained from medical records. Events will be adjudicated by a cardiologist blinded to the randomisation and categorised as cardiovascular (heart failure, AF, or other), non-cardiovascular, or of unknown cause.

\section{Statistical analysis}

Data analysis for efficacy will be performed according to the intention-to-treat principle for the assigned study groups. It is anticipated that randomisation of 70 patients will provide robust statistical power to detect plausible and clinically relevant improvement in $\mathrm{VO}_{2 \text { peak }}$ of $2 \mathrm{~mL} / \mathrm{min} /$ $\mathrm{kg}$ with a standard deviation of $3 \mathrm{~mL} / \mathrm{min} / \mathrm{kg}$ assuming $80 \%$ power and two-sided alpha level of 0.05 . This calculation is based on a prior randomised study in heart failure patients suggesting that iron deficient and replete individuals exhibit at least a clinically relevant $2 \mathrm{~mL} / \mathrm{min} /$ $\mathrm{kg}$ difference in $\mathrm{VO}_{2 \text { peak }}{ }^{37}$ To allow for a $15 \%$ discontinuation rate, 84 patients will be recruited, equating to 42 patients per group.

Continuous variables will be reported as mean and standard deviation, or median and interquartile range as appropriate. Categorical variables will be reported as number and percentage. The data will be checked for normality prior to the main analysis. Unpaired t-tests or Mann-Whitney $\mathrm{U}$ tests will be used to compare the changes in $\mathrm{VO} 2_{\text {peak }}$ between the two treatment arms, depending on the distribution of the data. Paired t-tests or Wilcoxon signed-rank tests will be used to evaluate the changes from baseline to week 12 in both treatment arms. All statistical tests will be two-sided, and a $\mathrm{p}$ value of $<0.05$ will be considered significant. All analyses will be undertaken using R V.4.0.2.

\section{Ethics and dissemination}

Ethics and governance approvals were obtained by the local Ethics Committee (Central Adelaide Local Health Network Committee, Australia; with additional acceptance of this approval by the University of Adelaide Human Research Ethics Committee). Written informed consent will be obtained from all study participants prior any study-specific assessments. The findings from this study will be disseminated through publications in peer-reviewed journals and national and international conferences.

\section{DISCUSSION}

This is the first study to examine the use of FCM in patients with $\mathrm{AF}$ and ID. The increasing burden of $\mathrm{AF}$ on individuals and healthcare systems warrants investigation into therapies that will improve the disease through novel mechanisms. Knowledge on the epidemiology and pathophysiology of ID in AF is still emerging. An investigation into the safety and efficacy of iron supplementation in these patients provides a logical starting point in this area. The results of this study will provide evidence on the importance of screening and correcting ID in patients with $\mathrm{AF}$.

Iron repletion has an established role in HFrEF patients. The large, multinational FAIR-HF (Ferinject Assessment in Patients with Iron Deficiency and Chronic Heart Failure) and CONFIRM-HF (Ferric Carboxymaltose Evaluation on Performance in Patients with Iron Deficiency in Combination with Chronic Heart Failure) trials demonstrated that iron repletion with intravenous FCM significantly improves symptoms, exercise tolerance and QoL in these patients. Importantly in FAIR-HF, this was irrespective of the presence of anaemia. Societal guidelines have since recommended iron testing on initial diagnosis of heart failure, as well as treatment of comorbid ID. ${ }^{38}$ Recently, the AFFIRM-AHF (A Randomised, Doubleblind Placebo Controlled Trial Comparing the Effect of Intravenous Ferric Carboxymaltose on Hospitalisations and Mortality in Iron Deficient Subjects Admitted for Acute Heart Failure) trial also demonstrated reduction in the risk of heart failure hospitalisations following iron repletion in HFrEF patients. ${ }^{31}$

Improving the symptomatology of AF and QoL is an important, but oft-neglected aspect of care. ID may be a common comorbidity in these patients and correcting the deficiency in other patient populations has been shown to improve the symptoms of fatigue and exercise tolerance. Beyond its intrinsic appeal, improvements in these symptoms may lead to improved engagement in risk factor management in patients with AF. The IRON-AF study began recruitment in July 2020 following delays due 
to the COVID-19 pandemic and is expected to complete recruitment by the end of 2021 .

Twitter Celine Gallagher @CelineGallaghe2, Ricardo S Mishima @Rmishima3 and Prashanthan Sanders @PrashSanders

Contributors CXW and PS were involved in conception and trial design. SJT and CXW were involved with drafting the manuscript. SJT, AE, NH-R, CG, RSM, EL, DW, NC, KCR-T, MS, ME, DL, PS and CXW were involved with trial conduct and data acquisition, critical revision of the manuscript for important intellectual content and final approval of the manuscript. Vifor Pharma, a part-funder of the study, was not involved with study design, and will not be involved with collection, management, analysis and interpretation of the study data. The decision to submit this protocol, and the future decision to submit the publication of the study data, is the responsibility of the investigators.

Funding The IRON-AF trial is an investigator-initiated study and the trial sponsor the University of Adelaide. Partial funding, including supplies of iron carboxymaltose, was provided by Vifor Pharma. CG is supported by a Postdoctoral Fellowship from the University of Adelaide (grant number: $N / A$ ). ME is supported by a Postgraduate Scholarship from the University of Adelaide (grant number: N/A). DL is supported by a Mid-Career Fellowship from the Hospital Research Foundation (grant number: N/A). PS is supported by a Practitioner Fellowship from the National Health and Medical Research Council of Australia (grant number: N/A) and by the National Heart Foundation of Australia (grant number: N/A). CXW is supported by a MidCareer Fellowship from the Hospital Research Foundation (grant number: N/A) and a Postdoctoral Fellowship from the National Heart Foundation of Australia (grant number: N/A)

Competing interests DL reports the University of Adelaide has received on his behalf lecture and/or consulting fees from Abbott Medical, Bayer, Biotronik, Boehringer Ingelheim, Medtronic, Microport and Pfizer/BMS. PS reports having served on the advisory board of Medtronic, Abbott Medical, Boston Scientific, CathRx and PaceMate. PS reports that the University of Adelaide has received on his behalf lecture and/or consulting fees from Medtronic, Abbott Medical and Boston Scientific. PS reports that the University of Adelaide has received on his behalf research funding from Medtronic, Abbott Medical, Boston Scientific and Microport. CXW reports that the University of Adelaide has received on his behalf lecture, travel and/or research funding from Abbott Medical, Bayer, Boehringer Ingelheim, Medtronic, Novartis, Servier, St Jude Medical and Vifor Pharma.

Patient and public involvement Patients and/or the public were not involved in the design, or conduct, or reporting, or dissemination plans of this research.

Patient consent for publication Not required.

Provenance and peer review Not commissioned; externally peer reviewed.

Open access This is an open access article distributed in accordance with the Creative Commons Attribution Non Commercial (CC BY-NC 4.0) license, which permits others to distribute, remix, adapt, build upon this work non-commercially, and license their derivative works on different terms, provided the original work is properly cited, appropriate credit is given, any changes made indicated, and the use is non-commercial. See: http://creativecommons.org/licenses/by-nc/4.0/.

\section{ORCID iDs}

Samuel J Tu http://orcid.org/0000-0003-4137-1978

Celine Gallagher http://orcid.org/0000-0002-5114-400X

Prashanthan Sanders http://orcid.org/0000-0003-3803-8429

Christopher X Wong http://orcid.org/0000-0002-1913-6675

\section{REFERENCES}

1 Wong CX, Brown A, Tse H-F, et al. Epidemiology of atrial fibrillation: the Australian and Asia-Pacific perspective. Heart Lung Circ 2017;26:870-9.

2 Gallagher C, Hendriks JM, Giles L, et al. Increasing trends in hospitalisations due to atrial fibrillation in Australia from 1993 to 2013. Heart 2019;105:1358-63.

3 Freeman JV, Wang Y, Akar J, et al. National trends in atrial fibrillation hospitalization, readmission, and mortality for Medicare beneficiaries, 1999-2013. Circulation 2017;135:1227-39.

4 Wong CX, Brooks AG, Leong DP, et al. The increasing burden of atrial fibrillation compared with heart failure and myocardial infarction: a 15-year study of all hospitalizations in Australia. Arch Intern Med 2012;172:739-41.
5 Wong CX, Brooks AG, Lau DH, et al. Factors associated with the epidemic of hospitalizations due to atrial fibrillation. Am J Cardiol 2012;110:1496-9.

6 Dorian P, Jung W, Newman D, et al. The impairment of healthrelated quality of life in patients with intermittent atrial fibrillation: implications for the assessment of investigational therapy. J Am Coll Cardiol 2000;36:1303-9.

7 Andrade J, Khairy P, Dobrev D, et al. The clinical profile and pathophysiology of atrial fibrillation: relationships among clinical features, epidemiology, and mechanisms. Circ Res 2014;114:1453-68.

8 Elshazly MB, Senn T, Wu Y, et al. Impact of atrial fibrillation on exercise capacity and mortality in heart failure with preserved ejection fraction: insights from cardiopulmonary stress testing. $J \mathrm{Am}$ Heart Assoc 2017;6. doi:10.1161/JAHA.117.006662. [Epub ahead of print: 31 Oct 2017].

9 Pardaens K, Van Cleemput J, Vanhaecke J, et al. Atrial fibrillation is associated with a lower exercise capacity in male chronic heart failure patients. Heart 1997;78:564-8.

10 Singh SN, Tang XC, Singh BN, et al. Quality of life and exercise performance in patients in sinus rhythm versus persistent atrial fibrillation: a Veterans Affairs Cooperative studies program substudy. J Am Coll Cardiol 2006;48:721-30.

11 Kato Y, Suzuki S, Uejima T, et al. The relationship between resting heart rate and peak VO2: a comparison of atrial fibrillation and sinus rhythm. Eur J Prev Cardiol 2016;23:1429-36.

12 Tsuneoka H, Koike A, Nagayama O, et al. Prognostic value of cardiopulmonary exercise testing in cardiac patients with atrial fibrillation. Int Heart $J$ 2012;53:102-7.

13 Wynn GJ, Todd DM, Webber M, et al. The European heart rhythm association symptom classification for atrial fibrillation: validation and improvement through a simple modification. Europace 2014:16:965-72.

14 Atwood JE, Myers JN, Tang XC, et al. Exercise capacity in atrial fibrillation: a substudy of the Sotalol-Amiodarone atrial fibrillation efficacy trial (SAFE-T). Am Heart J 2007;153:566-72.

15 Fiala M, Bulková V, kňouřil L. Functional improvement after successful catheter ablation for long-standing persistent atrial fibrillation. EP Europace 2016;19:1781-9.

16 Lau DH, Nattel S, Kalman JM, et al. Modifiable risk factors and atrial fibrillation. Circulation 2017:136:583-96.

17 Malmo V, Nes BM, Amundsen BH, et al. Aerobic interval training reduces the burden of atrial fibrillation in the short term: a randomized trial. Circulation 2016;133:466-73.

18 Donnellan E, Wazni OM, Harb S, et al. Higher baseline cardiorespiratory fitness is associated with lower arrhythmia recurrence and death after atrial fibrillation ablation. Heart Rhythm 2020;17:1687-93.

19 Pathak RK, Elliott A, Middeldorp ME. Impact of cardiorespiratory fitness on arrhythmia recurrence in obese individuals with atrial fibrillation: the CARDIO-FIT study. J Am Coll Cardiol 2015;66:996.

20 Garnvik LE, Malmo V, Janszky I, et al. Physical activity, cardiorespiratory fitness, and cardiovascular outcomes in individuals with atrial fibrillation: the HUNT study. Eur Heart J 2020;41:1467-75.

21 Beard J, Tobin B. Iron status and exercise. Am J Clin Nutr 2000;72:594S-7.

22 Brownlie T, Utermohlen V, Hinton PS, et al. Tissue iron deficiency without anemia impairs adaptation in endurance capacity after aerobic training in previously untrained women. Am J Clin Nutr 2004:79:437-43.

$23 \mathrm{Zhu}$ Yl, Haas JD. Altered metabolic response of iron-depleted nonanemic women during a 15-km time trial. J Appl Physiol 1998;84:1768-75.

24 Okonko DO, Mandal AKJ, Missouris CG, et al. Disordered iron homeostasis in chronic heart failure: prevalence, predictors, and relation to anemia, exercise capacity, and survival. J Am Coll Cardiol 2011:58:1241-51.

25 Jankowska EA, Rozentryt P, Witkowska A, et al. Iron deficiency predicts impaired exercise capacity in patients with systolic chronic heart failure. J Card Fail 2011:17:899-906.

26 Enjuanes C, Klip IT, Bruguera J, et al. Iron deficiency and healthrelated quality of life in chronic heart failure: results from a multicenter European study. Int J Cardiol 2014;174:268-75.

27 Comín-Colet J, Enjuanes C, González G, et al. Iron deficiency is a key determinant of health-related quality of life in patients with chronic heart failure regardless of anaemia status. Eur J Heart Fail 2013;15:1164-72.

28 Houston BL, Hurrie D, Graham J, et al. Efficacy of iron supplementation on fatigue and physical capacity in non-anaemic iron-deficient adults: a systematic review of randomised controlled trials. BMJ Open 2018;8:e019240. 
29 Anker SD, Comin Colet J, Filippatos G, et al. Ferric carboxymaltose in patients with heart failure and iron deficiency. $N$ Engl J Med 2009;361:2436-48.

30 Ponikowski P, van Veldhuisen DJ, Comin-Colet J, et al. Beneficial effects of long-term intravenous iron therapy with ferric carboxymaltose in patients with symptomatic heart failure and iron deficiency†. Eur Heart J 2015;36:657-68.

31 Ponikowski P, Kirwan B-A, Anker SD, et al. Ferric carboxymaltose for iron deficiency at discharge after acute heart failure: a multicentre, double-blind, randomised, controlled trial. Lancet 2020;396:1895-904.

32 Keskin M, Ural D, Altay S, et al. Iron deficiency and hematinic deficiencies in atrial fibrillation: a new insight into comorbidities. Turk Kardiyol Dern Ars 2018;46:103-10.

33 Harada M, Van Wagoner DR, Nattel S. Role of inflammation in atrial fibrillation pathophysiology and management. Circ $J$ 2015;79:495-502.

34 Tu SJ, Hanna-Rivero N, Elliott AD, et al. Associations of anemia with stroke, bleeding, and mortality in atrial fibrillation: a systematic review and meta-analysis. J Cardiovasc Electrophysiol 2021;32:686-94.
35 Abed HS, Wittert GA, Leong DP, et al. Effect of weight reduction and cardiometabolic risk factor management on symptom burden and severity in patients with atrial fibrillation: a randomized clinical trial. JAMA 2013;310:2050-60.

36 Wasserman K, Hansen JE, Sue DY. Measurements during integrative cardiopulmonary exercise testing. principles of exercise testing and interpretation: including pathophysiology and clinical applications. 5th ed. Philadelphia: Lippincott Williams and Wilkins, 2012: 71-106.

37 Okonko DO, Grzeslo A, Witkowski T, et al. Effect of intravenous iron sucrose on exercise tolerance in anemic and nonanemic patients with symptomatic chronic heart failure and iron deficiency FERRICHF: a randomized, controlled, observer-blinded trial. J Am Coll Cardiol 2008;51:103-12

38 Ponikowski P, Voors AA, Anker SD, et al. 2016 ESC guidelines for the diagnosis and treatment of acute and chronic heart failure: the task force for the diagnosis and treatment of acute and chronic heart failure of the European Society of cardiology (ESC). developed with the special contribution of the heart failure association (HFA) of the ESC. Eur J Heart Fail 2016;18:891-975. 